\title{
Correction for quadrature errors
}

\author{
Netterstrøm, A.; Christensen, Erik Lintz
}

Published in:

Proceedings of the International Geoscience and Remote Sensing Symposium

Link to article, DOI:

10.1109/IGARSS.1994.399297

Publication date:

1994

Document Version

Publisher's PDF, also known as Version of record

Link back to DTU Orbit

Citation $(A P A)$ :

Netterstrøm, A., \& Christensen, E. L. (1994). Correction for quadrature errors. In Proceedings of the International Geoscience and Remote Sensing Symposium: Surface and Atmospheric Remote Sensing: Technologies, Data Analysis and Interpretation (Vol. Volume 2, pp. 909-911). IEEE. https://doi.org/10.1109/IGARSS.1994.399297

\section{General rights}

Copyright and moral rights for the publications made accessible in the public portal are retained by the authors and/or other copyright owners and it is a condition of accessing publications that users recognise and abide by the legal requirements associated with these rights.

- Users may download and print one copy of any publication from the public portal for the purpose of private study or research.

- You may not further distribute the material or use it for any profit-making activity or commercial gain

- You may freely distribute the URL identifying the publication in the public portal 


\title{
Correction for Quadrature Errors
}

\author{
A.Netterstrøm, E. Lintz Christensen
}

Electromagnetics Institute, Technical University of Denmark

B-348, Lundtoftevej 100, DK-2800 Lyngby, Denmark; Fax +45 45931634

Phone +45 4288 14 44; E-mail an@emi.dtu.dk

\begin{abstract}
In high bandwidth radar systems it is necessary to use quadrature devices to convert the signal to/from baseband. Practical problems make it difficult to implement a perfect quadrature system. Channel imbalance and quadrature phase errors in the transmitter and the receiver result in error signals, which appear as self-clutter in the radar image. When digital techniques are used for generation and processing of the radar signal it is possible to reduce these error signals.
\end{abstract}

In this paper the quadrature devices are analyzed, and two different error compensation methods are considered. The practical implementation in the Danish polarimetric SAR system, EMISAR, is presented, and measured results will be given.

\section{INTRODUCTION}

Digital signal generation and processing is often used in pulse compression systems, where the ability to change the pulse modulation, bandwidth, and duration is required. One important issue in these systems is the conversion between the analog bandpass signal modulated on an intermediate frequency, and the complex digital baseband representation used in the data processing. In low bandwidth systems the conversion can be carried out by direct sampling or generation of the analog bandpass signal, and the bandpass to baseband conversion is implemented digitally. In systems, where the bandwidth is in the same order as the maximum conversion frequency of the $\mathrm{A} / \mathrm{D}$ and $\mathrm{D} / \mathrm{A}$ converters, the bandpass to baseband conversion must be implemented using analog quadrature modulators and demodulators.

Analog quadrature modulators and demodulators have practical problems. It is difficult to ensure that the transfer function for the real part of the baseband signal is the same as the transfer function for the imaginary part of the signal. As a result an error signal, called the image signal, is generated. Consider a signal having an offset frequency $f_{0}$, then the image signal will have the offset frequency $-f_{0}$ and an amplitude proportional to the complex difference between the two transfer functions. The image signal will not be compressed by the matched filter, but will cause small sidelobes, that degrade the integrated sidelobe ratio (ISLR). In fact, the image signal is often one of the more significant contributors to the degradation of the system ISLR.

When the new Danish polarimetric SAR sensor, EMISAR, [Christensen et al, 1994] was designed, the bandpass to baseband issue was examined carefully. The system uses a flexible digital pulse generation and data processing scheme, that enables correction or compensation for analog system deficiencies. It was decided to use bascband sampling and signal generation, and then implement a digital correction for the quadrature device deficiencies. Two correction methods were considered. The first method can be regarded as a correction by pre-distortion and equalizing. The method is a more general version of the method presented in [Churchill et al, 1981]. The method requires an accurate error model for the quadrature device. From this model the "inverse" signal processing can be derived. The processing is implemented in the digital system, and the combined processing of the quadrature device and the digital signal processing corresponds to a perfect device.

The second method is based on a principle presented in [Nichols et al, 1986]. The phase of the radar signal is incremented on each pulse. This is equivalent to modulating the radar signal to an offset frequency which is a fraction of the pulse repetition frequency. The desired signal will be centred on the positive offset frequency and the image signal will be centered on the negative offset frequency. If the pulse repetition frequency and the offset frequency are selected properly with respect to the spectrum of the signal, the spectrum of the desired signal and the image signal will not overlap, and hence it will be possible to remove the image signal by simple filtering.

In the following a complete analysis of the quadrature device deficiencies and the two correction methods will be given. The implementation in the EMISAR sensor will be presented, and finally performance measurements are given.

\section{TRANSFER FUNCTION REPRESENTATION}

A narrow band signal having the amplitude modulation a(t) and the phase modulation $\phi(t)$ is represented by the complex modulation function $\mathrm{z}(\mathrm{t})$ given by:

$z(t) \quad=\quad a(t) \exp (j \phi(t))=u(t)+j v(t)$

Here $u(t)$ and $v(t)$ are real signals, denoted the in-phase and the quadrature signal respectively.

Any system device can be regarded as a complex system which transforms a complex input to a complex output. A linear complex system can represent most system devices, but not the quadrature device. The quadrature device must be modelled as a linear real system having two real inputs, corresponding to the real and imaginary part of the complex input signal, and two real outputs corresponding to the real and imaginary part of the complex output signal.

The processing of the quadrature device can thus be expressed

$$
\begin{aligned}
& u_{y}(t)=h_{u u}(t) \otimes u_{x}(t)+h_{u v}(t) \otimes v_{x}(t) \\
& v_{y}(t)=h_{v u}(t) \otimes u_{x}(t)+h_{v v}(t) \otimes v_{x}(t)
\end{aligned}
$$

Where $u_{x}(t)$ and $v_{x}(t)$ are the input signals, $u_{y}(t)$ and $v_{y}(t)$ are the output signals, and $h_{u u}(t), h_{u v}(t) h_{v u}(t)$ and $h_{v v}(t)$ are the impulse responses of four real filters. $\otimes$ denotes convolution.

The process can also be written in the frequency domain by taking the Fouricr transform on cach side of equation. (2):

$$
\begin{aligned}
& U_{y}(f)=H_{u u}(f) U_{x}(f)+H_{u v}(f) V_{x}(f) \\
& V_{y}(f)=H_{v u}(f) U_{x}(f)+H_{v v}(f) V_{x}(f)
\end{aligned}
$$

Where $U_{y}(f), V_{y}(f)$ are the Fourier transform of the output signals, $U_{x}(f), V_{x}(f)$ are the Fourier transform of the input signals, and $H_{u}(f), H_{u v}(f), H_{v u}(f), H_{v y}(f)$ are the transfer functions of the four filters. 
Equation (3) can be written as a simple matrix multiplication:

$\mathbf{Y}(\mathrm{f})=\mathbf{H}(\mathrm{f}) \mathbf{X}(\mathrm{f})$,

where

$$
\begin{aligned}
& \mathbf{X}(f)=\left\{\begin{array}{l}
U_{x}(f) \\
V_{x}(f)
\end{array}\right\} \quad Y(f)=\left\{\begin{array}{l}
U_{y}(f) \\
V_{y}(f)
\end{array}\right\} \\
& \mathbf{H}(f)=\left\{\begin{array}{ll}
H_{u u}(f) & H_{u v}(f) \\
H_{v u}(f) & H_{v v}(f)
\end{array}\right\}
\end{aligned}
$$

We will define $\mathrm{H}$ as the transfer function matrix, define $\mathbf{X}$ and $\mathbf{Y}$ as signal vectors, representing the complex modulation functions of the in- and output signals.

Consider a real bandpass filter having the complex modulation function $\mathrm{Z}_{\mathrm{H}}(\mathrm{t}) \leftrightarrow \mathrm{Z}_{\mathrm{H}}(\mathrm{f})$ ( $\leftrightarrow$ denotes Fourier transform). The filtering is then described by:

$$
z_{y}(t)=z_{H}(t) / 2 \otimes z_{x}(t),
$$

Where $z_{x}(t)$ and $z_{y}(t)$ are the complex representation of the input and output signal respectively.

If we split $z_{H}(t)$ into real and imaginary parts $z_{H}(t)=n(t)+j m(t)$ and use eqn. (2)-(4), we can find the transfer function matrix for the bandpass filter:

$$
\mathbf{H}(f)=\left\{\begin{array}{cc}
N(f) & -M(f) \\
M(f) & N(f)
\end{array}\right\}
$$

Where $n(t) \leftrightarrow N(f)$ and $m(t) \leftrightarrow M(f)$.

It can be shown that for quadrature modulators and demodulators the transfer function matrix is not as simple, i.e. $H_{u u}(f) \neq H_{v v}(f)$ or $\mathrm{H}_{\mathrm{vu}}(\mathrm{f}) \neq-\mathrm{H}_{\mathrm{uv}}(\mathrm{f})$. The difference $\mathrm{H}_{u \mathrm{u}}(\mathrm{f})-\mathrm{H}_{\mathrm{vv}}(\mathrm{f})$ and the sum $\mathrm{H}_{\mathrm{vu}}(\mathrm{f})+\mathrm{H}_{\mathrm{uv}}(\mathrm{f})$ are a direct measure of the difference between the transfer function for the in-phase signal and the transfer function for the quadrature signal. We can rewrite the matrix expression:

$$
\begin{aligned}
\left\{\begin{array}{l}
U_{y}(f) \\
V_{y}(f)
\end{array}\right\} & =\left\{\begin{array}{ll}
H_{u u}(f) & H_{u v}(f) \\
H_{v u}(f) & H_{v v}(f)
\end{array}\right\}\left\{\begin{array}{l}
U_{x}(f) \\
V_{x}(f)
\end{array}\right\} \\
& =\frac{1}{2}\left\{\begin{array}{ll}
H_{u u}(f)+H_{v v}(f) & -\left(H_{v u}(f)-H_{u v}(f)\right) \\
H_{v u}(f)-H_{u v}(f) & H_{u u}(f)+H_{v v}(f)
\end{array}\right\}\left\{\begin{array}{l}
U_{x}(f) \\
V_{x}(f)
\end{array}\right\} \\
& +\frac{1}{2}\left\{\begin{array}{ll}
H_{u u}(f)-H_{v v}(f) & -\left(H_{v u}(f)+H_{u v}(f)\right) \\
H_{v u}(f)+H_{u v}(f) & H_{u u}(f)-H_{v v}(f)
\end{array}\right\}\left\{\begin{array}{c}
U_{x}(f) \\
-V_{x}(f)
\end{array}\right\}
\end{aligned}
$$

or written as a complex signal:

$$
z_{y}(t)=h_{\Sigma}(t) \otimes z_{x}(t)+h_{\Delta}(t) \otimes z_{x}^{*}(t)
$$

Where ${ }^{*}$ denotes complex conjugation.

We see that the output signal consists of two signals. One signal is the original modulation function passed through the filter having the complex impulse response $h_{\Sigma}(t)$. The other is the complex conjugated modulation function (the image signal) passed through the filter having the complex impulse response $h_{\Delta}(t)$. For a perfect modulator or demodulator $\left(h_{\Delta}(t)=0\right)$ the image signal will be zero.

\section{CORRECTION BY PRE-DISTORTION AND EQUALIZING}

In this section we will use the matrix description to derive a correction scheme. First we consider the radar transmitter. The transmitter consists of a signal generator and a number of devices (modulator, upconverter, filters etc.) which are represented by the transfer function matrix $\mathbf{A}$. The signal generator generates a signal represented by the vector $\mathbf{X}$, and hence the transmitted signal vector is AX. Assume the desired waveform is given by the vector $\mathbf{Y}$. This is achieved if the generated signal is pre-distorted so that $\mathbf{X}=$ $\mathbf{A}^{-1} \mathbf{Y}$.

First of all, the method requires that the matrix $\mathbf{A}^{-1}$ exists. This is always true if quadrature devices with small errors are consid ered. Secondly, it must be possible to generate the required wave- form. In modern systems, where the radar signal is generated digitally, it is possible to generate any bandlimited waveform. Finally, the method requires that we know the transmitter transfer function matrix very accurately. Many devices are easily measured using traditional techniques. Unfortunately, devices such as modulators and mixers are difficult to measure. It is therefore difficult to achieve a perfect correction.

The receiver converts the received signal into a digital baseband signal. The receiver consists of a downconverter, filters, demodulator, and A/D converters. These devices are represented by the transfer function matrix $\mathbf{B}$. The received echo signal at the receiver input is given by the vector $\mathbf{Y}$, and at the receiver output the signal vector is BY. We can correct for the receiver by equalizing the receiver output with transfer function matrix $\mathbf{B}^{-1}$. The corrected output is then $\mathbf{B}^{-1} \mathbf{B Y}=\mathbf{Y}$. The equalization can be combined with the matched filtering, but a more complex processing is required, since the equalization corresponds to two complex filterings, see equation (5).

Again the method requires that $\mathbf{B}^{-1}$ exists which is true if small errors are considered, and as for the transmitter it is also difficult to measure the transfer function matrix accurately.

It is important to recognize that the correction for the transmitter must be done using pre-distortion, and the correction for the receiver must be done by equalizing the received signal. If we for example pre-distorted the generated signal by $(\mathbf{B A})^{-1}$, we would obtain a perfect correction for a target at zero range delay without using equalizing. However, the correction would not work for target in a different range due to the different phase shift from transmitter to receiver.

\section{COMPENSATION BY PHASE TWIDDLING}

Now a different approach to overcome the problem is considered. Again the transmitter is represented by the transfer function matrix $\mathbf{A}$, and the receiver is represented by the matrix $\mathbf{B}$. For the moment we assume that the target is stationary at zero range delay, the overall matrix is $\mathbf{C =} \mathbf{B A}$. We further assume that the original pulse modulation is $\mathrm{x}(\mathrm{t})$ and that the pulse repetition frequency is $f_{\text {prf. }}$. We now phase shift the pulse by $\theta$ before pulse transmission, and by $-\theta$ after reception. Using equation (5) we find the resulting signal on the $n^{\prime}$ th transmission:

$y(n, t)$

$$
\begin{aligned}
& =\exp \left(-j \theta_{n}\right)\left(h_{\Sigma, C}(t) \otimes x(t) \exp \left(j \theta_{n}\right)+h_{\Delta, C}(t) \otimes\left(x(t) \exp \left(j \theta_{n}\right)\right)^{*}\right) \\
& =h_{\Sigma, C}(t) \otimes x(t)+h_{\Delta, C}(t) \otimes x(t) \exp \left(-j 4 n \pi f_{d} / f_{p r f}\right)
\end{aligned}
$$

Where $h_{\Sigma, C}(t)$ and $h_{\Delta, C}(t)$ are derived from $C$, and $\theta_{n}=$ $2 n \pi f / f$ prf is the phase used on the n'th pulse. The first term is the desired signal and the second term is the error signal caused by quadrature device deficiencies.

The Fourier transform of the pulse to pulse signal at a particular range delay $t_{0}\left(y\left(n, t_{0}\right)\right)$ consist of two $\delta$-functions. One at zero frequency corresponding to the desired signal, and one at $2 \mathrm{f}_{0}$ corresponding to the error signal. It follows that the error signal can be suppressed by simple lowpass filtering. The actual suppression is determined by the filter attenuation at $2 \mathrm{f}_{0}$.

If the target is not stationary the $\delta$-functions will widen, corresponding to the Doppler bandwidth B of the received signal. To ensure that the error signal can be separated from the desired signal we must select $f_{0}>2 B$, and $f_{\text {prf }}>B+2 f_{0}$. Therefore the pulse repetition frequency must be twice what is needed just to represent the Doppler bandwidth of the signal.

It can also be shown that this method suppresses baseband DC offset signals.

A difference between the pre-distortion/equalizing method, and the phase twiddle method is that pre-distortion/equalizing result in perfect signal processing i.e. error signals are not generated. The phase twiddle method only suppress the error signals after filtering in the receiver and this means that the error signals will be present in the system. This is not a problem if the error signals are small. Larger error signals may cause problems in nonlinear devices such as a TWT. 


\section{IMPLEMENTATION AND RESULTS}

In the EMISAR system pre-distortion can be implemented at no extra cost, since the hardware can generate the needed waveforms. The correction by equalizing would require two special filters, because of the motion compensation strategy used. The phase twiddle method could be implemented without extensions to the system. If the phase-twiddle method is combined with predistortion, an almost ideal correction is obtained. It was therefore decided to implement the phase twiddle method first, and to implement the pre-distortion if further improvement is needed.

Fig. 1 shows the relevant parts of the radar system. The code generator consists of four memory banks, each holding the desired signal, phase shifted $0,-\pi / 2,-\pi,-3 \pi / 2$. By selecting data from one of the four banks the signal phase is decremented by $-\pi / 2$ from pulse to pulse. The data are forwarded to two 8 bit D/A converters operating at $200 \mathrm{MHz}$ which generate the analog baseband signal The signal is then upconverted to the RF frequency by the Tx module.

The reflected signal is converted from the $R F$ frequency to baseband by the Rx module, and digitized by two 8 bit A/D operating at $100 \mathrm{MHz}$. After the peak data rate is reduced by a buffer, the signal is phase shifted digitally by $0, \pi / 2, \pi, 3 \pi / 2$ as required by the compensation method. Finally, the pulse to pulse filtering is implemented by the pre-filter. In the SAR system the digital phase shifter and the pre-filter were present for other purposes (motion compensation and data reduction), so no special hardware is needed for implementing the compensation.

To illustrate the method in practice a $20 \mu \mathrm{s}, 40 \mathrm{MHz}$ sine puise was generated and looped back into the receiver. Figure 2 shows the signal spectrum when no correction is used. The error signal at $-40 \mathrm{MHz}$ is $29 \mathrm{~dB}$ below the signal at $40 \mathrm{MHz}$, and the DC offset is $23 \mathrm{~dB}$ below the signal. Figure 3 shows the spectrum when phase twiddle compensation is used. It is seen that the image signal and the DC offset completely disappear in the noise. The remaining signals are interference picked-up by the analog parts of the system.

\section{CONCLUSION}

Two different methods for compensating for non-ideal quadrature devices have been investigated. This first method gives in theory an ideal compensation, but requires very accurate measurements that are difficult to obtain in practice. The other method is easy to implement, but requires a higher pulse repetition frequency than needed just to represent the radar signal. The second method has been implemented, and results shows that the compensation method works in practice.

\section{REFERENCES}

Churchill, F.E., Ogar, G.W., Thompsom, B.J. "The Correction of II and Q Errors in a Coherent Processor", IEEE Trans. Aeroap. Electron. Syst., vol AES-17, no. 1, Jan. 1981, pp 131-137.

Christensen, E.L., Madsen, S.N., Dall, J., Skou, N., Jørgensen,

J.H., Woelders, K., Netterstrøm, A., Granholm, J., Dich, M. "The Danish Polarimetric SAR for Remote Sensing

Applications", IGARSS '94, 1994'International Geoscience and

Remote Sensing Symposium, California, USA, 8-12 August 1994.

Nichols, A.D., Wilhelm, J.W., Gaffield, T.W., Inkster, D.R.,

Leung, S.K. "A SAR for Real-Time Ice Reconnaissance", IEEE Trans. Geosci. Remote Sensing, vol. GE-24, no. 3, May 1986 , pp. 383-389.

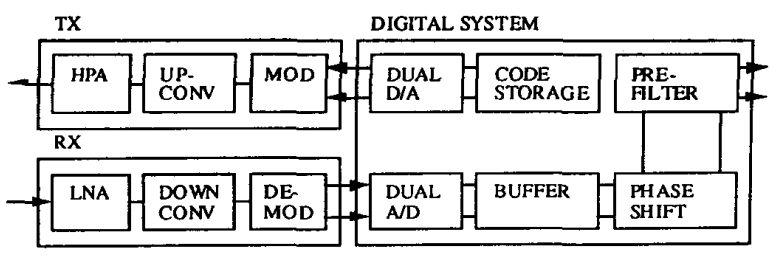

Fig. 1. Functional overview

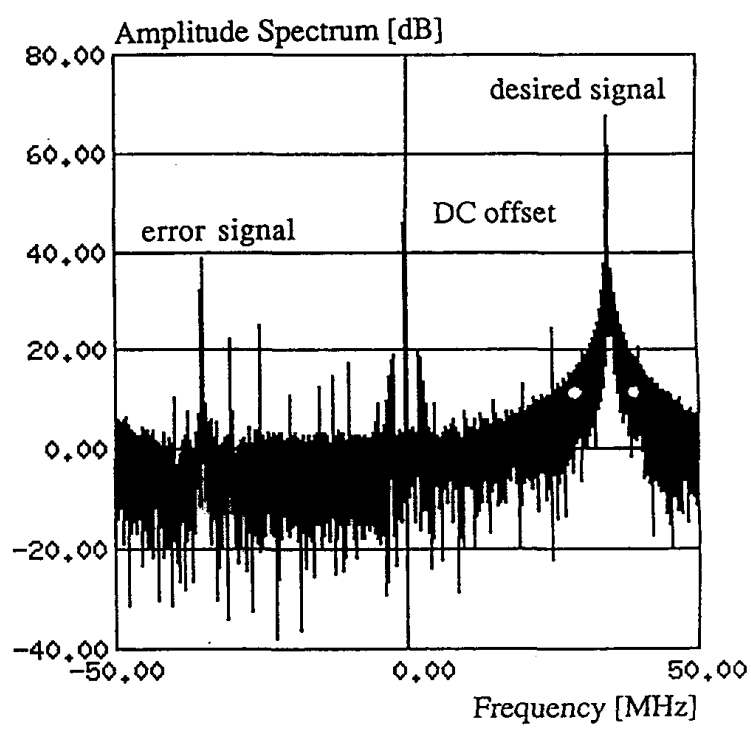

Fig. 2. Uncorrected test signal spectrum.

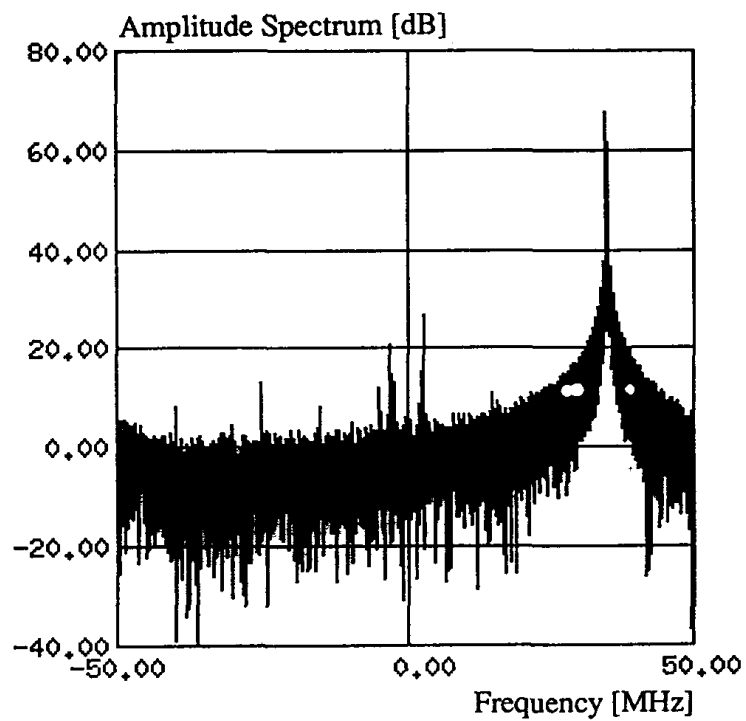

Fig. 3. Corrected test signal spectrum. 